\title{
DOKUMENTATION
}

\section{Das iranische Wahlgesetz zur Wahl der zweiten Majles 1984}

\author{
Von Ulrich Deffaa und Eberhard Krüger
}

Seit dem Sturz des Schah sind Informationen über das Rechtswesen des Iran nur schwer zugänglich. Lediglich die Verfassung der Islamischen Republik Iran von 1979 ist in westliche Sprachen übersetzt und im Ausland verbreitet worden. ${ }^{1}$ Andere Gesetzestexte sind nicht einmal im Original verfügbar, obwohl hierzulande durchaus ein Interesse an der Frage besteht, wie der islamische Anspruch auf dem Gebiet des Rechts in einem Land verwirklicht wird, das früher so stark auf die westlichen Industriestaaten ausgerichtet war wie gerade der Iran. Von besonderem Interesse ist hierbei das Wahlrecht, denn die Idee des durch Wahlen legitimierten Parlaments entstammt der aufgeklärten europäischen Staatstheorie und hat von dorther Eingang in die Staaten der islamischen Welt gefunden.

Das unter der Pahlavi-Dynastie geltende Wahlrecht für das Unterhaus bot Möglichkeiten für Wahlmanipulationen verschiedenster Art. ${ }^{2}$ So wurden auch diesbezügliche Vorwürfe unter dem Schah nach jeder Wahl erhoben. Die schwersten Mißstände sollten durch die Wahlrechtsreform von 1963 beseitigt werden: ${ }^{3}$ Arbeiter und Landpächter waren von nun an in den vom Provinzgouverneur berufenen Wahlausschüssen vertreten, Wahlen mußten am selben Tag im ganzen Land stattfinden, Wahllisten wurden eingeführt (wodurch eine wirksame Kontrolle der Stimmberechtigung erst möglich wurde) und Frauen erhielten das aktive und passive Wahlrecht. Nicht geändert wurde der Modus der Stimmabgabe (nicht Ankreuzen, sondern Niederschreiben der Namen), der Analphabeten und damit weite Kreise der Bevölkerung praktisch von der Stimmabgabe ausschloß. Auch blieb es weiterhin bei der Wahlprüfung durch das Parlament, also durch die Gewählten selbst und nicht durch ein unabhängiges Organ. Das neue Wahlrecht versucht, den Erfahrungen mit dem Wahlrecht der Schahzeit Rechnung zu tragen und zugleich die islamische Ordnung im Bereich des Wahlrechts verwirklichen. Es glie-

I Z. B. Verfassung der Islamischen Republik Iran, hg. von der Botschaft der Islamischen Republik Iran, Bonn 1980.

2 Vgl. dazu Kaviani, Das Problem demokratischer Wahlen im Iran, Tübingen (Jur. Diss.) 1963; dort S. 145-157 Ubersetzung der damals geltenden Wahlgesetze.

3 Vgl. Iran Almanac - 1972 and book of facts, publ. by Echo of Iran, Teheran o. J. S. 101; ferner Gehrke/Mehner (Hrsg.), Iran. Natur-Bevölkerung-Geschichte-Staat-Wirtschaft, Tübingen, Basel 1975, S. 187. 
dert sich in zehn Abschnitte: Allgemeines, Charakter der Wahlen, Ubertretungen und Vergehen, Voraussetzungen für das aktive und passive Wahlrecht, Wahlausschuß, Erklärung der Kandidatur, Wahlwerbung, Einsprüche und deren Prüfung, Strafen, Konstituierung und Eröffnung der Majles.

Die hier der Úbersetzung vorangestellten Erläuterungen wollen - soweit bei der gebotenen Kürze möglich - die aus Platzmangel nicht abgedruckten Bestimmungen des Gesetzes zusammenfassend darstellen, Charakteristika des Gesetzes aufzeigen und Hintergründe skizzieren.

\section{Wahlkreise}

Die Abgeordneten der Majles werden in Wahlkreisen gewählt. Die Einteilung der Wahlkreise folgt einem Mischsystem aus Territorial- und Personalprinzip: Der muslimische Anteil der Bevölkerung (etwa $98 \%)^{4}$ wählt in geographisch abgegrenzten Wahlkreisen, während die Wahlkreise der anerkannten (!) religiösen Minderheiten ${ }^{5}$ personell definiert sind. Die Einteilung der muslimischen Wahlkreise folgt der Verwaltungsgliederung des Iran, ${ }^{6}$ ein Wahlkreis entspricht einem Distrikt. Wegen der unterschiedlichen Größe der Distrikte ist auch die Anzahl der in jedem Wahlkreis zu wählenden Abgeordneten unterschiedlich. ${ }^{7}$ Hinsichtlich der Vorbereitung und Durchführung der Wahlen gibt es folgende organisatorische Ebenen: Im Bereich der Zentralregierung ist das Innenministerium für die Durchführung des Wahlgesetzes zuständig ( $\$ 25) .{ }^{8}$ Es erfüllt die zentralen Aufgaben wie Prüfung der Bewerber auf ihre Zulassungsvoraussetzungen ( $\S 47$, $50,51)$, Information der Offentlichkeit über alle Fragen der Wahl $(\S \S 15,23)$, Festsetzung der Offnungszeiten der Wahlräume (§ 11), sowie des Termins des zweiten Wahlgangs (§ 9) und gegebenenfalls des Termins für Nachwahlen ( 8 ). Es sammelt die Wahlergebnisse aus den einzelnen Wahlkreisen (\$ 24) und stellt schließlich die Beglaubigungsschreiben für die gewählten Abgeordneten aus $(\S \S 70,71)$. Den Provinzen fallen im Rahmen der Durchführung der Wahl keinerlei Aufgaben zu, die nächsten Instanzen unterhalb des Innenministeriums sind die Wahlausschüsse auf Distrikts- und die auf Unterdistriktsebene. Im Prinzip unterscheidet das Gesetz funktionell zwischen diesen auf zwei Ebenen angesiedelten Instanzen, doch sind Wahlausschuß des Wahlkreises und

4 Heine in: Ende/Steinbach, Der Islam in der Gegenwart. Entwicklung und Ausbreitung - Staat, Politik und Recht - Kultur und Religion, München 1984, S. 145.

5 Grds. 13 IranV; ferner unten "Religiöse Minderheiten".

6 Das Staatsgebiet des Iran gliedert sich in Provinzen, Distrikte und Unterdistrikte mit je einem Gouverneur an der Spitze. In einem Distrikt gibt es in der Regel ein städtisches Zentrum, das einen eigenen Unterdistrikt bildet ("Zentraler Unterdistrikt"). Ihm entspricht im Wahlrecht die "Zentrale des Wahlkreises". Die anderen, "ländlichen" Unterdistrikte umfassen jeweils mehrere dörfliche Siedlungen. Ausnahmen gelten für die großen Städte.

7 In Teheran sind beispielsweise 30 Abgeordnete zu wählen, vgl. IRNA (Islamic Republic News Agency) Bulletin $5 / 84$ v. 30. 4. (Bonn), S. 4.

$8 \S \S$ ohne nähere Angabe sind solche des Wahlgesetzes. 
Wahlausschuß der Zentrale des Wahlkreises institutionell verschmolzen: "Alle Angelegenheiten der Wahl im zentralen Unterdistrikt regelt der Wahlausschuß des Distrikts." (§ 45). Und: bei Aufgaben, die diesem "verschmolzenen" Wahlausschuß zufallen, wird im Verlauf des Gesetzes nicht mehr getrennt zwischen solchen, die ihm qua Distriktskompetenz und solchen, die ihm qua Unterdistriktskompetenz zufallen; unter der Bezeichnung "Wahlausschuß der Zentrale des Wahlkreises" werden ihm Aufgaben zugewiesen, die ihm nur in seiner Funktion als Wahlausschuß des Wahlkreises zukommen können. ${ }^{9}$ Die ländlichen Unterdistrikte werden, im Unterschied zum zentralen Unterdistrikt (Zentrale des Wahlkreises, Houze-e 'asli), als "Außenbereiche des Wahlkreises" (Houze-e far'i) bezeichnet. Ihre Zuständigkeiten sind beschränkt. ${ }^{10}$

\section{Wahlaufsicht, Vorkehrungen gegen Wahlmanipulationen}

Bei der Verhinderung von Wahlmanipulationen liegt einer der Schwerpunkte des Gesetzes, was vor dem Hintergrund der nicht endenden Klagen über Praktiken zur Schahzeit gesehen werden muß. Die Wahlprüfung obliegt jetzt einem institutionell unabhängigen Organ, dem "Wächterrat". " Er wählt die "Zentrale Aufsichtskommission" (§ 41). Diese erledigt gewissermaßen die "Alltagsarbeit" der Wahlaufsicht, ${ }^{12}$ während der Wächterrat die letzte Entscheidung über die Gültigkeit der Wahl hat. Er kann aber auch, sozusagen im Vorfeld, aus eigener Initiative Wahlausschüsse ablösen. Die Aufsichtskommissionen der Provinzen sind die einzigen auf dieser Ebene angesiedelten Wahlorgane. Da sie zudem nur in einem einzigen Fall zuständig sind, ${ }^{13}$ handelt es sich hier möglicherweise um eine Art "redaktionelles Versehen" des Gesetzgebers. Die Aufsichtskommissionen der Wahlkreise sind die wichtigsten Aufsichtsorgane bei der Wahl selbst. Sie werden bei praktisch allen Abweichungen vom vorgeschriebenen Ablauf tätig und überwachen die Wahlen "vor Ort" ${ }^{14}$ Aufsichtskommissionen auf Unterdistriktsebene erwähnt das Gesetz nicht.

9 Z. B. endgültige Zulassung der Kandidaten im Wahlkeis (§ 52); Auslosung zwischen stimmengleichen Kandidaten ( $\S 8$ Erl. 2); Erstellung des Protokolls des Wahlergebnisses ( $§ 26)$. Ähnliches gilt im Verhältnis von Gouverneur des Distriktes und Gouverneur des zentralen Unterdistriktes.

10 Bestimmung der Wahlräume ( $(38)$; Mitwirkung an der Wahl der Wahlvorstände für die einzelnen Wahlräume ( $(42)$; Entscheidung über den Einsatz mobiler Wahlräume (§ 46). Den Gouverneuren dieser Unterdistrikte fallen nur bedeutungslose Aufgaben zu.

11 Vgl. Grds. 99 IranV, ferner § 3. Der Wächterrat besteht aus 6 "gerechten islamischen Rechtsgelehrten“ und 6 "muslimischen Juristen verschiedener Rechtsgebiete" und soll "Widersprüche zwischen den Beschlüssen der Versammlung des Nationalrates und den islamischen Vorschriften der Verfassung verhindern", Grds. 91 IranV.

12 Entscheidung über Aufklärungssendungen zur Wahl im Fernsehen (§ 15); Sammlung der Bekanntmachungen der Wahlkreise ( $(41)$; Zustimmung zur Neuwahl von zentralen Wahlausschüssen für den 2 . Wahlgang ( $\$ 44$ Erl.); Zustimmung zur Ablösung einzelner Wahlausschußmitglieder oder ganzer Wahlausschüsse (54).

13 Behandlung von Beschwerden nicht zugelassener Bewerber ( $\$ 53$ ).

14 Mitwirkung bei der Aussonderung ungültiger Stimmen ( $\S 18,19)$; Aufsicht über die Vorbereitung der Wahlurnen (22); Anwesenheit bei der Wahl der Wahlausschüsse (34); Weiterleitung von Vorwürfen gegen Gouverneure (§ 36 Erl. 2); Begleitung der Urne in mobilen Wahlräumen (§ 46); Mitwirkung bei der Prüfung von Beschwerden $(\S 65)$. 
In den $\S \S 18-21$ finden sich detaillierte Regelungen zur Verhütung von Wahlmanipulationen. Stimmen, die in der dort genannten Weise abgegeben werden, sind ungültig; allerdings wird in den Bestimmungen darüber, wie mit solchen Stimmen zu verfahren ist, das Bemühen deutlich, die Zahl der nicht zu wertenden Stimmen so gering wie möglich zu halten. In einigen Fällen sollen die ungültigen Stimmen aussortiert werden, in anderen soll lediglich eine den ungültigen Stimmen entsprechende Anzahl von Stimmen durch das Los ausgeschieden werden. Es zeigt sich, daß die umfangreichen Listen unzulässiger Manipulationen vor allem den Charakter von Programmsätze haben. Wahlmanipulationen können deshalb nicht vollständig ausgeschlossen werden, weil Legitimität und Legalität nicht immer anhand des Stimmzettels festgestellt werden können: Einem Stimmzettel sieht man nicht an, ob die Stimme gekauft wurde (§18 Ziff. 2) oder ob sie mit einer falschen Kennkarte abgegeben wurde (§19 Ziff. 3).

\section{Wahlverfahren}

Die Stimmabgabe erfolgt auch heute noch durch Niederschreiben der Namen. Formal ist damit die Zugangsschwelle für Analphabeten unverändert hoch geblieben, die tatsächlichen Hindernisse dürften aber geringer geworden sein: Die Islamische Republik hat nach wie vor den stärksten Rückhalt unter der einfachen Bevölkerung, unter der der Analphabetismus noch am weitesten verbreitet ist. So wurde vor der Wahl die Teilnahme an ihr in den Massenmedien als religiöse Pflicht bezeichnet. Ferner erscheint die Möglichkeit der Einrichtung mobiler Wahlräume in entlegenen Gebieten (§ 46) nur dann sinnvoll, wenn auch in diesen Gebieten, wo der Analphabetismus sehr stark ist, eine gewisse Wahlbeteiligung zu erwarten ist. Die Islamische Republik war an einer hohen Wahlbeteiligung interessiert, weil die Wahl u. a. als Legitimation des gegenwärtigen Systems auch gegenüber dem Ausland angesehen wurde. ${ }^{16}$ Nicht bekannt ist den Verfassern allerdings, wie die Stimmabgabe durch Analphabeten geregelt war.

\section{Parteien}

Die Wahl ist eine reine Persönlichkeitswahl, es gibt keine Wahllisten: Parteien werden im Wahlgesetz mit keinem Wort erwähnt. In der Verfassung wird zwar ihre freie Gründung genau wie die anderer Vereine garantiert (Grds. 26 IranV), jedoch werden sie nicht im Zusammenhang mit Wahlen genannt: Antagonismen, als deren Verkörperung Parteien gelten, entsprechen nicht der islamischen Gesellschaftstheorie und Staatslehre. Parteien mit konkurrierenden Idealen würden die Einheit der islamischen Gemeinde

151976 waren $50 \%$ der über 14-jährigen Iraner Analphabeten, Fischer Weltalmanach 1984, Frankfurt a. M. 1983, S. 355.

16 Vgl. IRNA-Bulletin $4 / 84$ v. 18. 4. (Bonn), S. 5. 
stören. ${ }^{17}$ Demgemäß war auch 1984 keine Wahlwerbung z. B. im Namen der Partei der Islamischen Republik (IRP) zu beobachten. Nach einer Meldung der IRNA waren die meisten Kandidaten der Wahlen von 1984 parteilos; soweit sie einer Partei angehörten, war dies die IRP. ${ }^{18}$

\section{Wahlrecht}

Für das aktive Wahlrecht gelten nicht mehr die speziellen Beschränkungen der Schahzeit. So werden beispielsweise Bankrotteure, Bettler und Straftäter nicht mehr als vom aktiven Wahlrecht ausgeschlossen genannt. ${ }^{19}$ Weitergehenden Beschränkungen unterliegt das passive Wahlrecht. Nicht wählbar ist, wer weder in erkennbarer Weise dem Islam anhängt, insbesondere Apostaten, noch sich zu einer der anerkannten religiösen Minderheiten bekennt ( $\$ 30$ u. 32.2). Die Liste der mit einer Kandidatur unvereinbaren Funktionen ist umfangreich $(\S 30)$, ebenso die Liste der Verhaltensweisen, die eine Kandidatur ausschließen (§ 32).

\section{Stellung der Frau}

$\mathrm{Da}$ auch Frauen in der Islamischen Republik Iran das aktive und passive Wahlrecht zugestanden wurde, ist nicht als selbstverständlich anzusehen, wenn man einerseits bedenkt, daß sich die religiöse Agitation in den Unruhen von 1963 auch besonders gegen die seinerzeitige Einführung des Frauenwahlrechts richtete, ${ }^{20}$ und andererseits weiß, daß seit der islamischen Revolution von 1978/79 immer wieder von Versuchen berichtet wird, Frauen aus dem Berufsleben zu verdrängen. ${ }^{21}$ Ein Grundrecht auf Gleichberechtigung ist in der Verfassung nicht verankert, ${ }^{22}$ doch meldete die Nachrichtenagentur IRNA, ${ }^{23}$ auf einer Tagung über die Rolle der Frau im Islam im Oktober 1984 sei festgestellt worden, eine Zweitrangigkeit der Frau gebe es im Koran nicht, Unterschiede in der Rechtsstellung beträfen lediglich das Erbrecht, das Scheidungsrecht und den Zugang zum Richteramt.

17 Vgl. Binswanger, Das Selbstverständnis der Islamischen Republik Iran im Spiegel ihrer neuen Verfassung, in: Orient (Opladen) 21 (1980) S. 320 ff. (327).

18 IRNA-Bulletin $5 / 84$ v. 30.4 . (Bonn), S. 3.

19 So aber $\S 10$ des alten Unterhauswahlgesetzes, s. o. Fn. 2.

$20 \mathrm{Vgl}$. Keddie, Religion, Society, and Revolution in Modern Iran, in: Bonine/Keddie (eds.), Modern Iran. The Dialectics of Continuity and Change, Albany 1981, S. 32; ferner Steinbach in: Ende/Steinbach, a.a.O. (s. o. Fn. 4), S. 225.

21 Als Richterin tätig zu sein, wurde Frauen vom Justizministerium ausdrücklich untersagt, vgl. Steinbach a.a.O. (Fn. 20), S. 234. Angesichts dieser Tatsache ist es bemerkenswert, daß Frauen Mitglied der Legislative werden können.

22 Vgl. dazu die Grdse. 19-21 IranV; ferner Keddie, a.a.O. (Fn. 20), S. 34.

23 IRNA-Bulletin 19/84 v. 19. 11. (Bonn), S. 27. 


\section{Religiöse Minderheiten}

Da die Majles-Wahlen weder in der Verfassung (Grds. 62) noch im Wahlgesetz (§ 47) als "gleiche Wahlen" bezeichnet werden, ist es aus der Sicht des europäischen Juristen besonders interessant zu fragen, ob und nach welchen Kriterien Stimmen gegebenenfalls verschieden gewichtet werden. In einem nicht-säkularen islamischen Staat wäre am ehesten eine Diskriminierung nach Religionszugehörigkeit zu vermuten. Diese existiert tatsächlich; zwar haben alle iranischen Staatsbürger unabhängig von ihrer Religionszugehörigkeit das aktive Stimmrecht, jedoch bilden die Angehörigen der drei anerkannten religiösen Minderheiten (Juden, Christen, Zoroastrier; Grds. 13 IranV) eigene (in erster Linie) personell definierte Wahlkreise (§ 13). Ist nun dadurch eine Erfolgswertungsgleichheit der Stimmen zu Ungunsten dieser Minderheiten gegeben? Im Gegenteil: Während im Landesdurchschnitt ein Abgeordneter 148812 muslimische Bürger repräsentiert, ${ }^{24}$ entsenden jeweils 80000 Christen, ${ }^{25} 50000$ Juden $^{26}$ und 21000 Zoroastrier $^{27}$ einen Abgeordneten in die Majles.

\section{Strafen für Wahlverstöße}

Generell lassen sich bei den im Neunten Abschnitt angedrohten Strafen zwei Gruppen unterscheiden: Wahlverstöße von Privatpersonen, wozu auch Mitarbeiter der Presse gehören, werden in der Regel mit bis zu 74 Peitschenhieben, in schwereren Fällen mit Freiheitsstrafe bedroht. Werden hingegen Wahlverstöße von Personen begangen, die als Staatsbedienstete oder Mitglieder von Wahlinstanzen im Hinblick auf die Wahl besondere Verantwortung tragen, so werden diese nicht mit Peitschenhieben, sondern mit Gehaltskürzung, Dienstentfernung oder Ausschluß von den Wahlinstanzen für eine bestimmte Zeit, in schweren Fällen mit Freiheitsstrafen geahndet. Wichtig ist die Bestimmung, daß der Richter jede andere angemessene Strafe verhängen kann, die im islamischen Strafrecht, der Scharia, vorgesehen ist (§ 83).

24 Das methodische Problem bei dieser Berechnungsweise (Vergleich der Bevölkerungszahlen statt der Zahlen der Wahlberechtigten) ist nicht zu umgehen, da es keine Zahlen zu den Wahlberechtigten der verschiedenen Religionsgruppen gibt. Doch wird man davon ausgehen können, daß das Verhältnis der Zahl der Stimmberechtigten zur Gesamtzahl der jeweiligen Bevölkerungsgruppe in allen vier Religionsgemeinschaften in etwa gleich ist.

Zugrundegelegt wurden die Zahlen aus dem Fischer-Weltalmanach 1984 (Fn. 15), S. 355. Danach hat der Iran 40240000 Einwohner, wovon $98 \%=39435000$ Muslime sind. Auf sie entfallen 265 Abgeordnete.

253 Abgeordnete für 240000 Christen, Fischer-Weltalmanach, S. 355.

261 Abgeordneter für 50000 Juden, Fischer-Weltalmanach, S. 355.

271 Abgeordneter für 21000 Zoroastrier, Fischer-Weltalmanach, S. 355. 


\section{Die Wahlen 1984}

Die Wahlbeteiligung am ersten Wahlgang vom 15. 4. 1984 lag bei $70 \%{ }^{28}$ In diesem Wahlgang wurden 150 von 270 zu wählenden Abgeordneten mit der im ersten Wahlgang erforderlichen absoluten Mehrheit gewählt. ${ }^{29}$ Wegen des großen Andrangs mußte die Offnungszeit der Wahllokale verlängert werden. ${ }^{30}$ In manchen Distrikten wurden mobile Wahlräume eingesetzt. ${ }^{31}$ Am zweiten Wahlgang vom 17. 5. 1984 nahmen 16 Mio. Wähler teil, ${ }^{32}$ das entspräche einer Wahlbeteiligung von ca. $50 \%$. Dieser zweite Wahlgang war in 94 Wahlkreisen erforderlich. ${ }^{33}$ Wegen Unregelmäßigkeiten im Wahlverlauf wurde der Wahlvorgang in 12 Wahlkreisen abgebrochen ${ }^{34}$ Insgesamt wurden im ersten und zweiten Wahlgang 251 Abgeordnete gewählt. ${ }^{35}$ Die neue Legislaturperiode begann am 28. 5. 1984, am 5. 8.1984 waren immer noch erst 250 Sitze besetzt. ${ }^{36}$

Das Wahlgesetz wurde sehr spät verabschiedet, einen knappen Monat vor der Wahl. ${ }^{37}$ Das läßt darauf schließen, daß die Meinungen im Parlament über dieses Gesetz kontrovers waren. Der Gesetzestext selbst zeigt alle Anzeichen der Eile wie terminologische Uneinheitlichkeiten, teilweise unsystematischen Aufbau und Lückenhaftigkeit.

Ein Vergleich mit den Bestimmungen über die Wahl zur ersten Majles 1980 war leider nicht möglich, da diese nicht zur Verfügung standen.

28 IRNA-Bulletin $5 / 84$ v. 30.4 . (Bonn), S. 3.

29 IRNA-Bulletin $5 / 84$ ebd.

30 IRNA-Bulletin $4 / 84$ v. 18. 4. (Bonn), S. 3.

31 IRNA-Bulletin $4 / 84$ S. 4.

32 IRNA-Bulletin $7 / 84$ v. 1. 6. (Bonn), S. 12.

33 IRNA-Bulletin $7 / 84$ ebd.

34 IRNA-Bulletin $7 / 84$ ebd.

35 IRNA-Bulletin $7 / 84$, S. 13.

36 IRNA-Bulletin $13 / 84$ v. 15.8 . (Bonn), S. 5.

37 Dies ist vor allem deswegen problematisch, weil manche Bestimmungen des Gesetzes Fristen vorsehen, die 2 Monate vor der Wahl zu laufen begannen. 


\section{Verfassung und Recht in Übersee (Law and Politics in Africa, Asia and Latin America) - Beiheft 12}

Edited by Hamburger Gesellschaft für Völkerrecht und Auswärtige Politik

\section{Philip Kunig/ Wolfgang Benedek/Costa R. Mahalu Beiheft 12 Regional Protection Of Human Rights By Inter- national Law: The Emerging African System Documents and Three Introductory Essays}

The African Charter on Human Rights and Peoples' Rights was signed in 1981 and has not yet become binding under international law. Apart from its formal status at law, the Charter has already assumed considerable importance in the political dynamics of human rights in Africa. It has been conceived with regard to experience with universal and regional instruments for the international protection of human rights and has also successfully combined ideals which originally took shape in Europe and the Americas with traditions and values peculiar to African societies.

The present publication opens with an introduction by Dr. Costa Ricky Mahalu, University of Dar es Salaam, on »Africa and Human Rights «. Dr. Philip Kunig presents a comparison of the system of human rights protection under the 1981 charter with the systems established under the only two regional precursors that have operated with a measure of succes - those of Western Europe and the Americas. Dr. Wolfgang Benedek, University of Graz, offers an analysis of two salient features of the African charter, i. e. the collective ("peoples' «) rights and »individual duties « covered by the instrument. The authors would like to provide, through their contributions, a basis for appreciation of this African charter of human rights, thus perhaps also enabling it to receive more attention politically.

A document section includes the text of the Charter, a Memorandum of the Drafters, the 1979 Draft of the Charter, and the European and American Convention on Human Rights of 1950 resp. 1969.

1985, 156 S., 29,- DM, ISBN 3-7890-1126-6

Nomos Verlagsgesellschaft

Postfach $610 \cdot 7570$ Baden-Baden 


\section{Wahlgesetz zur Wahl der 2. Majlis (Auszüge)}

Jumhuri-ye Eslami, Jg. 5 Nr. 1394, 27. Esfand 1362 (= 18. März 1984)

\section{Erster Abschnitt: Allgemeines}

$\S 1$ Die Wahlen zur Islamischen Ratsversammlung erfolgen nach den Bestimmungen dieses Gesetzes. Die Regierung hat bei der Durchführung der Wahlen Sorge zu tragen, daß die Wahlen vor Beendigung der laufenden Legislaturperiode durchgeführt werden, damit das Land zu keiner Zeit ohne Versammlung ist.

Erläuterung: Die Bezeichnung "Versammlung" in diesem Gesetz steht für die Islamische Ratsversammlung.

§ 2 Die Zahl der Abgeordneten der Islamischen Ratsversammlung beträgt 270.

Erläuterung 1: Von der Gesamtzahl der Abgeordneten stehen 5 den religiösen Minderheiten zu, und zwar wie folgt: Je ein Abgeordneter den Zoroastriern und den Juden, den assyrischen und chaldäischen Christen zusammen ein Abgeordneter, den armenischen Christen des Südens und des Nordens je ein Abgeordneter.

Erläuterung 2: Das Verzeichnis der Wahlkreise für die zweite Legislaturperiode entspricht dem der ersten Legislaturperiode.

Erläuterung 3: Alle 10 Jahre, gerechnet von der Konstituierung der ersten Islamischen Ratsversammlung an, erhält turnusmäßig jeder Wahlkreis, in dem laut Volkszählungsstatistik die Bevölkerung um 150000 Personen gestiegen ist, einen weiteren Abgeordneten.

$\S 3$ Die Aufsicht über die Wahlen ist Aufgabe des Wächterrates. Diese Aufsicht ist umfassend und betrifft in allen Phasen sämtliche Angelegenheiten, die mit den laufenden Wahlen verbunden sind.

§ 4 Solange mehr als 6 Monate bis zum Ende der Legislaturperiode verbleiben, finden in vakanten Wahlkreisen Ersatzwahlen statt. Falls die Zahl der Abgeordneten vor Beendigung der Legislaturperiode unter 200 sinkt, werden vorgezogene Wahlen durchgeführt. $\S 5$ Die leitenden Beamten des Innenministeriums, der Provinzregierung und - mit Bestellung durch den Innenminister der leitende Beamte des Distrikts oder Unterdistrikts - handeln beim Vollzug dieses Gesetzes in Vertretung des Innenministers respektive des Gouverneurs der Provinz, des Distrikts oder Unterdistrikts.

$\S 6$ Bediensteten des Staates, staatlicher, angegliederter oder kommunaler Organisationen, Gesellschaften und Anstalten wird im Falle ihrer Wahl und der Aushändigung der Beglaubigungsurkunde die Zeit als Abgeordneter auf ihre Dienstzeit bezüglich Dienstalter und Dienstgrad angerechnet. Für die Dauer ihres Mandats erhalten sie lediglich Abgeordnetendiäten. 


\section{Zweiter Abschnitt: Charakter der Wahlen}

$\S 7$ Die Wahlen sind unmittelbar, allgemein und geheim.

$\S 8$ Die Wahl der Abgeordneten erfolgt im ersten Wahlgang mit absoluter Mehrheit der abgegebenen Stimmen, im zweiten Wahlgang mit relativer Mehrheit.

Erläuterung 1: Bei Stimmengleichheit zweier oder mehrerer Kandidaten entscheidet das Los, das in gemeinsamer Sitzung des Wahlausschusses der Zentrale des Wahlkreises und der Aufsichtsführenden des Wächterrates gezogen wird.

Erläuterung 2: In Wahlkreisen, wo Wahlen abgebrochen oder für ungültig erklärt wurden, und in vakanten Wahlkreisen, hat das Innenministerium im Einvernehmen mit dem Wächterrat binnen höchstens 2 Wochen erneut Wahlen durchzuführen.

§ 9 Ergibt sich im ersten Wahlgang für einen oder mehrere Kandidaten keine absolute Mehrheit, so erfolgt ein zweiter Wahlgang. An diesem nehmen von den Kandidaten, die unter der absoluten Mehrheit geblieben sind, diejenigen mit dem höchsten Stimmenanteil teil, insgesamt jedoch nur doppelt so viele, wie (noch) Abgeordnete zu wählen sind. Ist das Verhältnis der verbliebenen Kandidaten geringer als 2:1, so kandidieren alle von ihnen im zweiten Wahlgang.

Erläuterung 1: Entspricht die Zahl der verbliebenen Kandidaten der Zahl der zu ermittelnden Abgeordneten oder bleibt sie darunter, so entfällt ein zweiter Wahlgang, und die Stimmen des ersten Wahlganges entscheiden.

Erläuterung 2: Keiner der Bewerber um einen Abgeordnetensitz kann sich in mehr als einem Wahlkreis bewerben. Anderenfalls wird seine Kandidatur insgesamt hinfällig, und er wird von der Teilnahme an den Wahlen zu dieser Versammlung ausgeschlossen.

Erläuterung 3: Das Innenministerium bestimmt und verkündet im Einvernehmen mit dem Wächterrat den Zeitpunkt des zweiten Wahlganges im Verlaufe eines Monats nach Bekanntgabe der Ergebnisse des ersten Wahlganges und der Bestätigung der Korrektheit der Wahl durch den Wächterrat.

Erläuterung 4: Alle Stimmabgaben im zweiten Wahlgang erfolgen ausschließlich in dem Wahlkreis, in dem der Wähler am ersten Wahlgang teilgenommen hat.

$\S 10$ Bei jedem Wahlgang kann jede die Voraussetzungen erfüllende Person nur einmal unter Vorlage der Kennkarte mit Paßbild seine Stimme abgeben.

Erläuterung: In Fällen, wo eine Kennkarte mit Paßbild nicht vorhanden ist, muß ein Mitglied des Wahlvorstands unter Angabe seines Namens auf dem Personalienabschnitt des Stimmzettels vermerken, daß der Wähler wohlbekannt ist.

$\S 11$ Die Stimmabgabe erfolgt im gesamten Land am selben Tag und dauert zumindest 10 Stunden. Diese Frist kann bei Notwendigkeit verlängert werden.

Erläuterung 1: Dem Innenminister obliegt es, die Notwendigkeit der Verlängerung der Wahldauer in einem Wahlkreis oder im ganzen Land festzustellen.

Erläuterung 2: Die Stimmabgabe hat an einem öffentlichen Feiertage stattzufinden.

$\S 12$ Auf Anordnung des Innenministeriums, der Distrikts- oder Unterdistriktsgouverneure haben alle Ministerien, Organisationen, staatliche Verwaltungen und angegliederte Anstalten sowie die Gruppierungen der Revolution ihr Personal und sonstige 
Einrichtungen bis zum Abschluß der Wahlen zur Verfügung zu stellen. Wie sich versteht, ist der Zeitaufwand der besagten Bediensteten auf ihre Dienstzeit anzurechnen.

Erläuterung: Auf Anordnung des Wächterrates oder der von ihm eingesetzten Kommissionen haben alle genannten Gruppierungen und Organe ihre Bediensteten zur Durchführung der Wahlaufsicht zur Verfügung zu stellen. Ebenso haben das Innenministerium sowie die Provinz-, Distrikts- und Unterdistriktsgouverneure die notwendigen Einrichtungen zur Verfügung zu stellen.

$\S 13$ Die Wahlen der religiösen Minderheiten der Zoroastrier, Juden, Assyrer, Chaldäer und Armenier des Nordens finden zentral im Wahlkreis des Distrikts Teheran, für die Armenier des Südens finden sie zentral im Wahlkreis des Distrikts Esfahan statt, vermittelt durch die Gouverneure der Distrikte und Unterdistrikte, in denen die genannten Minderheiten ihren Wohnsitz haben.

$\S 14$ Falls gleichzeitig mit der Abhaltung von Wahlen zur Versammlung weitere Wahlen stattfinden, sind auf Anweisung des Innenministeriums für beide Wahlen Wahlräume mit gemeinsamen Ausschüssen und getrennten Wahlurnen vorzusehen.

$\S 15$ Das Fernsehen der Islamischen Republik Iran ist verpflichtet, Aufklärungsprogramme über die Wahlen, welche das Innenministerium oder die vom Wächterrat gewählte zentrale Aufsichtskommission für notwendig erklärt, und ebenso Verlautbarungen und Informationen zu den Wahlen im Einvernehmen mit der Direktion des Fernsehens landesweit oder über Regionalnetze des Fernsehens der Islamischen Republik Iran auszustrahlen.

$\S 16$ Die Wahlen zur zweiten Islamischen Ratsversammlung in den Wahlkreisen Abadan, Khorramshahr, Dasht-e Azadegan, Qasr-e Shirin, Mehran und Dehloran werden bis zur Wiederherstellung normaler Bedingungen für Wahlen und der Rückkehr der Flüchtlinge an die Stammsitze der genannten Wahlkreise im gesamten Land durchgeführt, vermittelt durch die Distrikts- und Unterdistriktsgouverneure.

Erläuterung 1: Die Stimmabgabe für die Kandidaten der Kriegsgebiete erfolgt unter Vorlage des Ausweises der "Stiftung für die Flüchtlinge des Aufgezwungenen Krieges" und bei Fehlen der Kennkarte allein anhand des beglaubigten Ausweises der Stiftung. Erläuterung 2: Flüchtlinge mit Kennkarte, die für die Kandidaten der genannten Gebiete stimmen wollen, haben sich den Ausweis der Stiftung zu beschaffen.

Erläuterung 3: Der Wahlvorstand hat Kennkarte und Ausweis mit einem Stempel zu versehen und den Ausweis zu kennzeichnen.

Erläuterung 4: Kombattanten, Zivilisten und wer immer sich in den besagten Gebieten aufhält, wählt, wie die übrige Bevölkerung mittels Kennkarte.

§ 17 Die Ordnungskräfte haben im Rahmen der Gesetze für Ordnung zu sorgen und jegliche Unregelmäßigkeit beim Ablauf der Wahlen und bei der Aufbewahrung der Urnen zu verhindern. Ordnungskräfte haben kein Recht, sich in Angelegenheiten der Aufsicht und Durchführung der Wahlen einzumischen.

$\S 18$ In den folgenden Fällen werden nach Bestätigung durch die Aufsichtskommission des Wahlkreises ungültige Stimmzettel zwar als abgegebene Stimmen gezählt, diese Stimmzettel jedoch unter Angabe des Sachverhalts dem Protokoll beigefügt: 
1. Unleserliche Stimmzettel,

2. Durch Kauf und Verkauf erlangte Stimmen,

3. Stimmzettel, die mit Namen des Stimmberechtigten oder mit Unterschrift bzw. Fingerabdruck versehen sind,

4. Stimmzettel, die nur Namen anderer als der zugelassenen Kandidaten enthalten.

Erläuterung: In Wahlkreisen, in denen mehr als ein Abgeordneter zu wählen ist, sind lediglich die unleserlichen Namen ungültig.

$\S 19$ In den folgenden Fällen werden nach Bestätigung durch die Aufsichtskommission des Wahlkreises ungültige Stimmzettel nicht als abgegebene Stimmen gezählt und besagte Stimmzettel unter Angabe des Sachverhalts dem Protokoll angeführt:

1. Bei Urnen, an denen Versiegelung oder Wahlsiegel fehlen,

2. Bei über die Zahl der Personalienabschnitte der Stimmzettel hinausgehenden Stimmen,

3. Bei Stimmen von Personen, welche das gesetzliche Wahlalter nicht erreicht haben,

4. Bei Stimmen, die mit Kennkarten von Verstorbenen oder Nichtiranern abgegeben wurden,

5. Bei Stimmen, welche durch Verfälschung oder Manipulation (im Personalienabschnitt, bei Stimmabgabe, Zählung oder Protokollierung) zustande kamen,

6. Bei Stimmen, die mit Kennkarten anderer Personen oder gefälschten Kennkarten abgegeben wurden,

7. Bei mehrfacher Stimmabgabe,

8. Bei Stimmen, die mit Kennkarten abwesender Personen abgegeben wurden,

9. Bei Stimmzetteln ohne Wahlstempel,

10. Bei weiß abgegebenen Stimmzetteln,

11. Bei durch Drohung erlangten Stimmen,

12. Bei Stimmen, die auf anderen Papieren als dem Stimmzettel abgegeben wurden.

Erläuterung 1: Alle Stimmen, die im Protokoll aufgeführt sind, und zu denen Wahlzettel in den Urnen oder Personalienabschnitte fehlen, sind ungültig und werden als nicht abgegeben angesehen.

Erläuterung 2: Die unter Ziffer 2 angeführten überzähligen Stimmen werden nach dem Losverfahren aus der Gesamtheit der Stimmen ausgesondert.

$\S 20$ Falls auf dem Stimmzettel über die Namen zugelassener Kandidaten hinaus weitere Namen eingetragen sind, ist der Stimmzettel nicht ungültig, nur die zusätzlichen Namen werden nicht berücksichtigt.

Erläuterung: Falls der Name eines Kanidaten mehrfach eingetragen wurde, wird ihm nur eine Stimme zugerechnet.

$\S 21$ Falls die eingetragenen Namen die erforderliche Zahl übersteigen, werden die überzähligen Stimmen vom letzten an nicht berücksichtigt.

$\S 22$ Vor Beginn der Stimmabgabe müssen in Anwesenheit der Vertreter der Aufsichtskommission für den betreffenden Wahlkreis die Urnen, die leer sein müssen, verschlossen, versiegelt und mit dem Siegel der Aufsichtskommission und des Distrikts bzw. Unterdistrikts des Wahlkreises versehen werden. In einem vor dem Abstimmungs- 
zeitraum im Wahllokal erstellten Protokoll bezeugen die Vertreter der Aufsichtskommission des betreffenden Wahlkreises die Anzahl der Urnen und ihren leeren Zustand. Falls im Verlauf der Stimmabgabe die Einführung einer weiteren Urne notwendig wird, wird in gleicher Weise vorgegangen und dieser Vorgang protokolliert.

$\S 23$ Das Innenministerium hat während des Zeitraums der Abhaltung von Wahlen in Erfüllung der ihm übertragenen Aufgaben die Offentlichkeit über die Wahlen betreffende Fragen aufzuklären.

$\S 24$ Die Distrikts- oder Unterdistriktsgouverneure der Zentralen des Wahlkreises haben nach Beendigung der Wahlen die Resultate ohne Verzögerung dem Innenministerium zur Veröffentlichung durch die Massenmedien mitzuteilen und danach die Ergebnisse im ganzen Wahlkreis bekanntzugeben.

$\S 25$ Das Innenministerium ist mit der Durchführung des Gesetzes zur Wahl der Islamischen Ratsversammlung betraut und verantwortlich für den geordneten Ablauf der Wahlen. Zu diesem Zweck kann es zur Kontrolle des Wahlablaufs Bevollmächtigte in die Wahlkreise und Wahllokale entsenden.

$\S 26$ Nach Beendigung der Wahlen wird das Protokoll des Wahlergebnisses, unterzeichnet vom Wahlausschuß des Zentrums des Wahlkreises und der Aufsichtskommission des betreffenden Wahlkreises, fünffach angefertigt. Eine Ausfertigung verbleibt beim zentralen Wahlausschuß, die anderen werden der genannten Aufsichtskommission, dem Innenministerium (2 Exemplare) und der zentralen Wahlaufsichtskommission übersandt.

\section{Dritter Abschnitt: Ưbertretungen und Vergehen}

$\S 27$ Die Begehung folgender Handlungen stellt eine strafbare Handlung dar:

1. Kauf oder Verkauf von Stimmen,

2. Manipulation oder Fälschen von Personalienabschnitten der Stimmzettel, von Stimmen oder Protokollen,

3. Bedrohung oder Verlockung in Wahlsachen,

4. Stimmabgabe mit gefälschter Kennkarte,

5. Stimmabgabe mit Kennkarte einer anderen Person,

6. mehrfache Stimmabgabe,

7. Störung des Wahlablaufs,

8. Verringerung oder Vermehrung (der Anzahl) der Stimmzettel oder der Personalienabschnitte der Stimmzettel,

9. Manipulation bei Stimmabgabe oder Auszählung,

10. Stimmenannahme auf die Kennkarte von Abwesenden,

11. Empfehlung durch Mitglieder des Wahlvorstandes, auf dem Stimmzettel den Namen eines bestimmten Kandidaten einzutragen, 
12. Abändern, Umändern, Fälschen, Entwenden oder Vernichten von Schriftstücken und Wahlakten wie Personalienabschnitten zum Stimmzettel, Stimmzettel, Protokollen, Fernschreiben, Telegrammen oder Telegraphien,

13. Offnen oder Erbrechen des verschlossenen Aufbewahrungsraumes, von Siegellack und Siegel der Wahlurnen ohne gesetzliche Ermächtigung,

14. Austausch, Veränderung oder Vernichtung von Wahlakten ohne Berechtigung,

15. Einwirkung auf den Wahlgang durch verfälschte Wahlakten,

16. Erzeugung von Furcht bei oder Einschüchterung der Abstimmenden oder des Wahlvorstandes beim Wahlgang, bewaffnet oder unbewaff net,

17. Einwirkung auf den Wahlvorgang durch Amtsanmaßung oder auf irgendeine ungesetzliche Weise.

Erläuterung: Falls dadurch, daß Ubertretungen im Sinne des obigen Paragraphen begangen wurden, der Ablauf der Wahl in einem oder mehreren Wahllokalen vom gesetzlich vorgesehenen Verlauf abweicht und das Gesamtergebnis der Wahlen beeinflußt, wird der Sachverhalt durch das Innenministerium der zentralen Aufsichtskommission zur Vorlage beim Wächterrat mitgeteilt.

$\S 28$ Die Einrichtungen der Justiz in jedem Wahlkreis ergreifen im Einvernehmen mit den Aufsichtsführenden des Wächterrates und des Wahlausschußes im Rahmen der einschlägigen Bestimmungen die notwendigen Schritte zur Verhütung von Straftaten.

\section{Vierter Abschnitt: Voraussetzungen für das aktive und passive Wahlrecht}

§ 29 Für die aktive Wahl müssen folgende Voraussetzungen gegeben sein:

1. Staatsangehörigkeit der Islamischen Republik Iran

2. Erreichen eines Alters von 16 Jahren

3. Geistige Zurechnungsfähigkeit

$\S 30$ Für die passive Wahl müssen folgende Voraussetzungen für die Zulassung gegeben sein:

1. Uberzeugung und Verpflichtung des Handelns auf den Islam,

2. Staatsangehörigkeit der Islamischen Republik Iran,

3. Uberzeugung und Verpflichtung des Handelns auf die Ordnung der Islamischen Republik Iran

4. Treuebekenntnis zur Verfassung

5. ausreichende Lese- und Schreibfähigkeit

6. Unversehrtheit des Seh-, Hör- und Sprechvermögens, soweit erforderlich

7. Erreichen eines Alters von mindestens 26 und höchstens 75 vollen Jahren.

Erläuterung: Bewerber um Sitze der in der Verfassung aufgeführten religiösen Minderheiten sind von der Voraussetzung in Ziffer 1 ausgenommen. Sie müssen eine feste Uberzeugung in ihrem eigenen Glauben haben.

$\S 31$ Folgende Personen sind mit Rücksicht auf ihr Amt und ihre Tätigkeit von der Kandidatur ausgeschlossen: 
1. Der Präsident der Republik und seine Berater, Premierminister und Minister mit ihren Beratern und Staatssekretären, Mitglieder des Wächterrates, Mitglieder des obersten Rates der Justiz, der Vorsitzende des Hofes für Verwaltungsgerechtigkeit, der Vorsitzende der Generalinspektion sowie alle Wehrbediensteten im ganzen Land, es sei denn, sie haben zwei Monate vor der Zulassung ihre Dienstgeschäfte aufgegeben und üben keinerlei Funktion in ihrer Stellung aus,

2. Imame auf Dauer an Freitagsmoscheen, Staatsanwälte, Hilfsstaatsanwälte, religiöse Richter, Provinzgouverneure mit ihren Beratern und Sachgebietsleitern, Ministerialdirektoren und Abteilungsleiter, Distrikts- und Unterdistriktsgouverneure, Leiter der Büros für islamische Propaganda und des Aufbaufeldzuges im gesamten Land und die Direktoren von Rundfunk und Fernsehen der Islamischen Republik Iran, es sei denn, sie haben zwei Monate vor der Bewerbung ihre Dienstgeschäfte aufgegeben und üben keinerlei Funktion in ihrer Stellung aus.

Erläuterung 1: Die in Ziffer 1. und 2. dieses Paragraphen festgelegte Frist beträgt für den ersten Wahlgang der zweiten Wahlperiode der Versammlung einen Monat,

Erläuterung 2: Wo nach dem Dienstrecht oder dem Anstellungsvertrag die Freistellung abhängig ist von der Zustimmung des Vorgesetzten, ist diese Zustimmung Bedingung. 3. Mitglieder von Wahlausschüssen und Aufsichtsführende des Wächterrates im Wahlkreis ihres Verantwortungsbereiches.

§ 32 Folgende Personen sind von der Kandidatur zur Versammlung ausgeschlossen:

1. Personen, die bei der Festigung der Grundlagen des verflossenen Regimes eine aktive und wirksame Rolle gespielt haben,

2. Großgrundbesitzer, die unbebauten Boden auf ihren Namen registrieren ließen,

3. Funktionäre von Parteien, Organisationen und Gruppierungen, deren Illegalität von den zuständigen Behörden festgestellt wurde,

4. Personen, die wegen einer Straftat gegen die Islamische Republik Iran verurteilt wurden,

5. wer durch Urteil der zuständigen Organe wegen Apostasie verurteilt ist,

6. wer wegen Unmoral allgemein bekannt oder notorisch ausschweifend ist,

7. zu einer Hadd-Strafe Verurteilte, es sei denn ihre Reue steht fest,

8. Rauschgiftsüchtige,

9. nicht-rechtsfähige Personen und solche, die von Grundsatz 49 der Verfassung betroffen sind. 


\section{Fünfter Abschnitt: Wahlausschuß}

\section{Sechster Abschnitt: Erklärung der Bewerbung}

$\S 50$ Das Innenministerium erstellt bei Eingehen der Angaben zu den Bewerbern von den Distrikts- und Unterdistriktsgouverneuren fortlaufend eine vollständige Liste und übersendet sie zur Úberprüfung früherer Vorgänge, welche die in diesem Gesetz gegebenen Zulassungsvoraussetzungen berühren, an die zuständigen Organe der "Dokumentationszentrale der Islamischen Revolution", "Informationsabteilung des Korps der islamischen Revolutionswächter", "Komitee der Islamischen Revolution", Generalstaatsanwaltschaft, des "Zentralamts für das Meldewesen" sowie des "Erkennungsdienstes und der Interpol«. Die Zentralen haben ihre Stellungnahmen binnen 48 Stunden mit Begründung und Unterlagen dem Innenministerium zu unterbreiten.

Erläuterung 1: Nach Schaffung des Informationsministeriums hat sich das Innenministerium Kenntnisse über frühere Vorgänge auf dem Amtswege über dieses Ministerium zu verschaffen.

Erläuterung 2: Das Innenministerium kann bei Erfordernis Fingerabdrücke von Bewerbern um einen Abgeordnetensitz nehmen.

$\S 52$ Die Wahlausschüsse der Zentralen der Wahlkreise haben, innerhalb von höchstens fünf Tagen vom Ablauf der Zulassungsfrist an, auf Grund örtlich notwendiger Prüfungen und unter Berücksichtigung der vom Innenministerium mitgeteilten Ergebnisse die Erfüllung der in diesem Gesetz gegebenen Zulassungsvoraussetzungen festzustellen und, soweit die gesetzlichen Voraussetzungen gegeben sind, die Kandidaten zu benennen, die an den Wahlen im Wahlkreis teilnehmen.

$\S 53$ Die Distrikts- und Unterdistriktsgouverneure der Zentralen der Wahlkreise haben die vom Wahlausschuß bestätigten Namenslisten binnen eines Tages bekanntzugeben und auf schnellstem Wege der Aufsichtskommission der Provinz zur Kenntnis zu bringen.

Erläuterung 1: Personen, für deren Zulassung eine Bestätigung nicht erfolgte, haben innerhalb von vier Tagen ab dem Datum der Veröffentlichung der Bekanntmachung das Recht, unter Angaben von Gründen Einspruch bei der Aufsichtskommission der Provinz einzulegen.

Erläuterung 2: Eingelangte Berichte und Einsprüche werden in der Sitzung der Aufsichtskommission der Provinz nach Ablauf der Einspruchsfrist binnen fünf Tagen geprüft und das Ergebnis in einem Protokoll dem Distrikts- oder Unterdistriktsgouverneur der Zentrale des Wahlkreises zur endgültigen Bekanntgabe mitgeteilt. 


\section{Siebenter Abschnitt: (Wahl-) Werbung}

§ 56 Die Wahlwerbung ist eröffnet mit der Bekanntgabe der Namen der Kandidaten. Sie endet 24 Stunden vor dem Tage der Stimmenabgabe.

$\S 57$ Niemand ist berechtigt, Bekanntmachungen und Werbeanschläge von Wahlbewerbern, welche an zulässiger Stelle angebracht sind, während der gesetzlichen Frist der Wahlwerbung zu zerstören oder zu beseitigen. Die Zuwiderhandlung gilt als Straftat. § 58 Die Durchführung jeglicher Art von Wahlwerbern vom Zeitpunkt der Bekanntgabe der Kandidaten an, sei es für oder gegen Kandidaten für einen Sitz in der "Versammlung", ist über Rundfunk und Fernsehen, vom Redepult des Freitagsgebets oder mit jeglichem anderen Mittel staatlichen oder offiziellen Charakters untersagt, ebenso Aktivitäten von Bediensteten während der Dienstzeit unter Benutzung von Einrichtungen und anderen Hilfsmitteln, von Ministerien und Abteilungen, von Gesellschaften und angegliederten Anstalten des Staats, von Gruppierungen und Anstalten, die aus dem öffentlichen Haushalt - in welcher Höhe auch immer - gespeist werden sowie das zur Verfügungstellen der genannten Einrichtungen und Hilfsmittel. Die Zuwiderhandlung gilt als Straftat.

Erläuterung 1: Anstalten und Gruppierungen, deren Vermögen öffentliches Gut ist, wie die "Stiftung für die Beraubten", sind in obigen Paragraphen eingeschlossen.

Erläuterung 2: Behörden, Organisationen, staatliche Organe und Gruppierungen sowie deren Mitarbeiter sind nicht berechtigt, unter Angabe ihres Amtes Verlautbarungen oder Bekanntmachungen für oder gegen einen Kandidaten herauszugeben oder zu plakatieren.

§59 Wahlausschüsse und die Wahlaufsicht sind nicht berechtigt, für oder gegen irgendeinen Wahlbewerber werbend tätig zu werden.

$\S 60$ Das Anbringen von Verlautbarungen, Bildern, Plakaten und jeglicher Art von Werbebekanntmachungen auf Wegweisern und Verkehrszeichen, Schildern von Krankenhäusern, Schulen und anderen staatlichen oder angegliederten Lehranstalten ist untersagt. Die Ordnungskräfte nehmen bei Wahrnehmung solcher Vorgänge die Täter fest und überstellen sie zur gesetzlichen Verfolgung den gerichtlichen Organen. Distrikt- und Unterdistriktgouverneure haben zur Beseitigung solcher Anschläge Schritte zu unternehmen.

$\S 61$ Jegliche Bekanntmachung oder Wahlwerbung am Wahlraum muß vor Beginn der Stimmabgabe durch den Wahlvorstand beseitigt werden.

$\S 62$ Presseorgane und Publikationen sind ab drei Tagen vor der Stimmabgabe nicht berechtigt, Anzeigen oder gegen Wahlwerber gerichtete Artikel aufzunehmen oder Artikel zu verfassen, welche die Ablehnung einzelner Kandidaten nahelegen. Davon abgesehen sind die Kandidaten berechtigt, innerhalb von 18 Stunden nach der Veröffentlichung der betreffenden Zeitung durch den Distrikts- oder Unterdistriktsgouverneur eine Gegendarstellung zugehen zu lassen. Diese hat die Zeitung entsprechend dem Pressegesetz unverzüglich zu veröffentlichen. Erscheint die Publikation vor der Bannfrist für Wahlwerbung nicht mehr, muß der Verantwortliche auf eigene Kosten die Gegendarstellung 
einer Zeitung oder Zeitschrift übersenden, die vor der Bannfrist erscheint, und deren Herausgeber hat sie in die nächstfolgende Ausgabe aufzunehmen. Auch in anderen als Presseerzeugnissen ist in der Frist von drei Tagen vor der Stimmabgabe die Veröffentlichung derartiger Beiträge untersagt. Vor der Bannfrist ist der betroffene Kandidat berechtigt, seine Meinung zu veröffentlichen.

$\S 63$ Bewerbern um einen Sitz und ihren Anhängern ist es in keiner Weise gestattet, negative Werbung gegen andere Bewerber zu betreiben. Ausschließlich die eigene Würdigkeit oder die Unterstützung eines Bewerbers sind darzulegen. Beleidigung und üble Nachrede der Bewerber ist jedermann untersagt und Übertretungen werden entsprechend den Bestimmungen bestraft.

$\S 64$ Ab 24 Stunden vor der Stimmabgabe bis zu deren Abschluß ist jegliche Wahlwerbung untersagt.

§ 65 Die Wahlausschüsse haben Einsprüche, die vom Tag der endgültigen Verlautbarung über die Zulassung der Bewerber bis zwei Tage nach Bekanntgabe des Wahlergebnisses eingehen, entgegenzunehmen und sie vom Tage des Einspruchs bis sieben Tage nach dem Wahlgange in gemeinsamer Sitzung des Wahlausschusses mit den Aufsichtsführenden des Wächterrats im Wahlkreis zu prüfen.

Erläuterung 1: Einsprüche, die die Form der Durchführung der Wahlen betreffen, können innerhalb von sieben Tagen nach dem Wahlgang unter Angabe der Gründe auch beim Sekretariat des Wächterrats eingereicht werden.

Erläuterung 2: Zur Prüfung der Einsprüche sind diese mit Angaben zu dem oder den Einspruchführenden, enthaltend Eigenname, Familienname, Namen des Vaters, Beruf, vollständige Anschrift, gegebenenfalls Fernsprechnummer sowie der Unterschrift des Einspruchführenden zu versehen.

Erläuterung 3: Falls der Einspruchsführer grundlose und unbelegte Beschuldigungen aus persönlichen Motiven gegen den Bewerber erhebt und das Vorgehen des Einspruchführers den Charakter der Verleumdung hat, so wird er dafür belangt.

Erläuterung 4: Das Vorbringen von Einsprüchen in vertraulicher Form sowie Prüfung und Offentlichmachen derselben ist untersagt.

$\S 66$ Einsprüche, welche im Verlauf der Wahlen bei den Wahlausschüssen eingelegt werden, unterbrechen den Fortgang der Wahlen nicht. Sie werden gleichzeitig mit den anderen Einsprüchen während der Prüfungsfrist behandelt.

$\S 67$ Falls der Wahlausschuß nach Prüfung der Einsprüche und Berichte feststellt, daß die Wahlhandlung in einem oder mehreren Wahlräumen vom normalen Ablauf abwich und die Wahl nicht korrekt durchgeführt wurde, erklärt er nach Bestätigung durch die Aufsichtsführenden des Wächterrats die Wahlen in dem oder den Wahlräumen für nichtig, wenn dies das Wahlergebnis nicht beeinflußt.

$\S 68$ Alle Einsprüche gegen die Wahl werden vor der Ubersendung der Wahlniederschrift an die "Versammlung" vom Innenministerium der zentralen Aufsichtskommission zugestellt. In gleicher Weise werden sie nach Ubersendung der Wahlniederschrift der "Versammlung" zugestellt.

Erläuterung: Der Wächterrat und die Aufsichtskommission haben alle Wahleinsprüche 
nach Ausstellung der Beglaubigungsschreiben an die "Versammlung" zu übersenden. $\S 69$ Falls die Uberprüfung der Einsprüche aus einzelnen Wahlkreisen zur Ungültigerklärung der Wahlen führt, ist es die Befugnis des Wächterrats, dies durch die Massenmedien bekanntzugeben.

§ 70 Die Ausstellung der Beglaubigungsschreiben der Gewählten ist abhängig von der Bestätigung der Korrektheit der Wahlen durch den Wächterrat. Dieser befindet darüber in kürzest möglicher Frist. Das Innenministerium stellt nach der Bestätigung der Korrektheit der Wahlen die Beglaubigungsschreiben unverzüglich aus.

$\S 71$ Die Beglaubigungsschreiben der Gewählten werden auf Weisung des Innenministeriums vom Wahlausschuß und den Aufsichtsführenden des Wächterrats mit Stempel und Unterschrift in vier Ausfertigungen ausgestellt.

\section{Achter Abschnitt: Strafen}

Neunter Abschnitt: Konstituierung und Eröffnung der Islamischen Ratsversammlung 